(aggression, PTSD, mental health treatment, SuD, childhood victimisation, lack of parental/caregiver support, intimate partner violence, free or reduced lunch (ie, SES proxy) were entered into a Robust Poisson model $(p \leq 0.25)$ to identify factors associated with having symptoms. Race did not meet criteria for model entry.

Results/Outcomes (1) 154 of 275 (56\%) offenders reported ongoing TBI-related symptoms. (2) SuD and PTSD were associated with having symptoms. An interaction was found between SuD and PTSD $(p=0.0236)$. (3) An association was further found between females with PTSD and TBI-related symptoms among those without $\mathrm{SuD}$ (adjusted prevalence ratio $(\mathrm{APR})=2.58$; CI 1.49 to $4.45, \mathrm{p}=0.0006)$, but not among those with $\mathrm{SuD} \quad(\mathrm{APR}=1.30 ; \mathrm{CI} 0.98$ to $1.73, \mathrm{p}=0.0662)$. (4) Compared to having neither condition, the APR for the association between $\mathrm{SuD}$ and TBI-related symptoms was 2.32 (CI 1.34 to 4.01). The APR for the association with TBI-related symptoms among offenders with both SuD and PTSD, compared to having neither, was 3.03 (CI 1.80 to 5.08).

Significance/Contribution to the Field PTSD and SuD are closely associated with ongoing TBI-related symptoms. Treating and managing all three conditions concurrently, as well as prevention efforts, would likely be cost effective and benefit both the female offenders and society.

\section{FACTORS ASSOCIATED WITH ONGOING TRAUMATIC BRAIN INJURY-RELATED SYMPTOMS AMONG FEMALE OFFENDERS}

doi:10.1136/injuryprev-2012-040580g.23

E Pickelsimer*, P Ferguson, M Cornelius. Medical University of South Carolina,

Division of Biostatistics \& Epidemiology, Charleston, South Carolina, USA

Background Ongoing traumatic brain injury (TBI)-related symptoms, post-traumatic stress disorder (PTSD), and substance dependency (SuD) can greatly affect an offender's ability to function while incarcerated and upon community reentry.

Aims/Objectives/Purpose To identify factors associated with ongoing TBI-related symptoms among female offenders.

Methods 275 female offenders were asked whether they experienced any of 19 ongoing symptoms after TBI. Variables 